\title{
Evaluation of reward processes in an animal model of depression
}

\author{
David A. Slattery • Athina Markou • John F. Cryan
}

Received: 7 September 2006 / Accepted: 25 October 2006 / Published online: 20 December 2006

(C) Springer-Verlag 2006

\begin{abstract}
Rationale Anhedonia is a core symptom of major depression. Deficits in reward function, which underlie anhedonia, can be readily assessed in animals. Therefore, anhedonia may serve as an endophenotype for understanding the neural circuitry and molecular pathways underlying depression.

Objective Surprisingly, there is scant knowledge regarding alterations in brain reward function after olfactory bulbectomy (OB), an animal model which results in a behavioural syndrome responsive to chronic antidepressant treatment. Therefore, the present studies aimed to assess reward function after bulbectomy.

Materials and methods The present study utilized sucrose preference, cocaine-induced hyperlocomotion and intracranial self-stimulation (ICSS) responding to examine reward processes in the OB model.
\end{abstract}

D. A. Slattery · J. F. Cryan

Neuroscience Research,

Novartis Institutes for BioMedical Research,

Novartis Pharma AG,

Basel, Switzerland

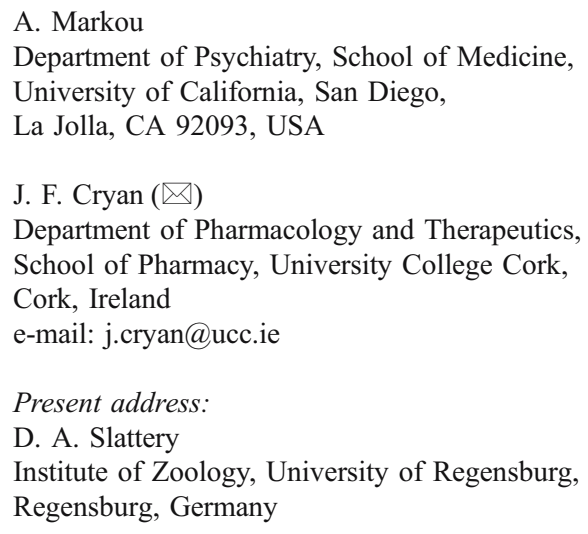

Results Bulbectomized animals showed a marked preference $(>90 \%)$ for $0.8 \%$ sucrose solution compared with water; similar to the preference exhibited by sham controls. Importantly, there were pronounced deficits in brain reward function, as assessed using ICSS, which lasted 8 days before returning to baseline levels. Furthermore, bulbectomized animals were hyper-responsive to the locomotor stimulating properties of an acute and a repeated cocaine regimen. However, no difference in ICSS facilitation was observed in response to an acute cocaine injection.

Conclusions Taken together, these results suggest that bulbectomized rats display alterations in brain reward function, but these changes are not long-lasting and thus, not amenable to investigating the effects of pharmacological interventions. However, given that $\mathrm{OB}$ animals are hypersensitive to drugs of abuse, bulbectomy may be an appropriate inducing factor for the development of animal models of co-morbid depression and drug dependence.

Keywords Animal model · Reward · Depression · Antidepressant

Affective disorders represent some of the most common and proliferating health problems worldwide. Major depression is predicted to become the second most common cause of global disease burden by 2020 (Murray and Lopez 1997), and lifetime prevalence rates are approximately $15 \%$ (Kessler et al. 2005). Unlike many diseases with clearly defined symptomology, major depression is a heterogeneous disorder, classified based on a cluster of symptoms described by the Diagnostic and Statistic Manual of Mental Disorders (DSM-IV) and International Classification of Diseases (ICD10). Therefore, there have been considerable clinical and preclinical research efforts to determine the underlying pathophysiology of depression to gain better insight into 
the disease. Of central importance to this approach is the availability of valid preclinical animal models for evaluation of the potential utility of novel pharmacotherapies.

The wide spectra of disruptions that characterize depression highlight the difficulty posing researchers to mimic the disorder in the laboratory. Nonetheless, numerous attempts have been made to create rodent models of depression, or at least model aspects of depression that are expressed as physiological and behavioural responses. Despite major differences in brain anatomy between rodents and humans, there are evolutionary conserved circuits between species, which underlie certain physiological and behavioural responses (Cryan and Holmes 2005). Thus, largely through inference, we can study these responses to elucidate behaviours and the neural circuits and genetic factors subserving them as a means to use lower species to understand human behaviour and disease. More recently, it has become clear that a useful strategy might be to model single endophenotypic differences (i.e. one clearly defined behavioural output) relevant to the disease state as opposed to a syndrome (Cryan and Holmes 2005; Cryan and Mombereau 2004; Gould and Gottesman 2006). Endophenotypes can be neuropsychological, cognitive, neurophysiological, neuroanatomical or biochemical in nature. In parallel, similar efforts have been made for dissecting various psychiatric disorders into specific endophenotypes (Gould and Gottesman 2006; Hasler et al. 2004). These more discrete clinical features potentially provide a more effective approach to identifying the genetic and neurobiological underpinnings of human disease and represent more tractable entities to model aspects of psychiatric disorders in animals.

Anhedonia, or the loss of pleasure or interest in previously rewarding stimuli, is one of the core symptoms of major depression. Moreover, it is one of the few symptoms which can be probed in animals, and therefore, can serve as an appropriate endophenotype for understanding the neural circuitry and molecular pathways underlying the disease. The mesolimbic dopamine brain circuit is known to be a major component of the brain reward system (Kornetsky 2004; Nestler 2005; Wise 2002), and dysfunctions of this system contributes to anhedonia (Markou et al. 1998). Although there has not been substantial research of the brain reward system in depression, a number of recent studies have used psychostimulants as a method to probe this system. These studies demonstrate a hypersensitivity of reward processes to an acute low dose of psychostimulant in depressed patients (Naranjo et al. 2001; Tremblay et al. 2002, 2005).

To develop a model of any endophenotype, an appropriate inducing factor and a robust behavioural measure are required (Geyer and Markou 2000). A number of techniques exist to study behavioural consequences of alterations in reward circuits preclinically, but paramount among these is intracranial self-stimulation (ICSS). The utility of ICSS is based on a number of facts: (1) There is no satiation or tolerance to the stimulation; (2) responding remains stable for long periods of time and (3) it by-passes sensory inputs (Kornetsky 2004; Markou and Koob 1992; Wise 2005). A number of brain regions that support ICSS responding in rodents have also been demonstrated in human neuroimaging studies to have altered activity in major depressed patients i.e. the hippocampus (Sheline et al. 2002; van der Kooy et al. 1977) and amygdala (Drevets 2001; Touzani and Velley 1998). Therefore, ICSS responding may represent a useful behavioural measurement of the brain reward function with which to study depression preclinically.

The bilateral surgical removal of the olfactory bulbs results in a constellation of behavioural, neurochemical and neuroendocrine changes that are selectively reversed by chronic, but not acute, antidepressant treatments (Cryan et al. 1998, 1999; Harkin et al. 2003; van der Stelt et al. 2005). This model has one of the best preclinical profiles for assessing the effects of novel antidepressant agents (Cryan et al. 2002). Therefore, the similarity of the deficits observed and the timeframe of their reversal with that of humans make the olfactory bulbectomy (OB) model very attractive for further elucidation of the pathophysiology of depression. Surprisingly, there is scant knowledge regarding alterations in the brain reward system after OB. The most robust behavioural phenotype observed after bulbectomy is hyperactivity in a novel environment (see Song and Leonard 2005 for review). Therefore, given that locomotor activity is dependent on dopaminergic activity (Kuczenski et al. 1983), it is suggestive of an altered dopaminergic system after bulbectomy, which in turn may affect brain reward function.

Therefore, the aims of the present studies were to promote a greater understanding of the brain reward system in the OB model of depression. To achieve this goal, we utilized three behavioural paradigms: sucrose preference, intracranial self-stimulation (ICSS) and the hyperlocomotor response to acute and repeated psychostimulant (i.e. cocaine) administration in the rat. These procedures enable assessment of the brain reward system under both basal and stimulated conditions. Furthermore, the use of ICSS, which does not require intact sensory inputs, enables study of the reward system in the bulbectomized rat without any possible negating influence of the loss of olfaction. Finally, we assessed the psychomotor and ICSS-facilitating response to a challenge dose of cocaine in bulbectomized and sham-operated rats to assess the response of these animals to a drug of abuse. It has been hypothesized that psychomotor stimulant properties of drugs, such as cocaine, indirectly reflect reward processes of these drugs (Wise and Bozarth 1982, 1985). Our hypothesis is that there would be deficits in reward function in the OB model. 


\section{Materials and methods}

Animals

Male Sprague-Dawley rats (Charles River, France) weighing between 270-350 $\mathrm{g}$ at the time of surgery were used in these studies. The animals were housed in groups of two or four and maintained on a 12-h light/dark cycle (lights on 6:00 A.M.) in a temperature-controlled colony $\left(22-24^{\circ} \mathrm{C}\right)$. The animals had free access to food and water. Animals were allowed to habituate for at least 7 days before surgery. All experimental procedures were subject to institutional review and conducted in accordance with the Veterinary Authority of Basel-Stadt, Switzerland.

\section{Olfactory bulbectomy}

Bilateral olfactory bulbectomy was carried out under isoflurane anaesthesia (1-3\% isoflurane, oxygen flow rate 450-500 CCM). Pre-operative analgesia (buprenorphine $0.1 \mathrm{mg} / \mathrm{kg}$, s.c.) was administered $30 \mathrm{~min}$ before surgery. After anaesthesia, a midline incision was made from approximately $1 \mathrm{~cm}$ posterior to $1 \mathrm{~cm}$ anterior to bregma. Burr holes, $\sim 2 \mathrm{~mm}$ in diameter, were then carefully drilled through the skull at approximately $7 \mathrm{~mm}$ anterior to bregma and $2 \mathrm{~mm}$ on either side of the midline. The olfactory bulbs were then gently removed by suction with care taken not to damage the cortex. Finally, the burr holes were filled with haemostatic sponge and the wound closed with surgical thread under aseptic conditions. Antiseptic liquid was applied liberally to the wound, and then the animals were returned to clean cages. Sham animals were treated in a similar manner; however, the bulbs were not aspirated. After surgery, the rats were returned to their home-cage; no cage contained two bulbectomized rats to minimize any enhanced intermale aggression that can arise (Kelly et al. 1997). Animals were housed in cages of 2-4 after surgery. The animals were given 14 days to recover from surgery before the start of behavioural testing and were handled and weighed daily to eliminate aggressiveness (Kelly et al. 1997).

\section{Sucrose preference}

After 14 days of recovery, animals were transferred into single housing with free access to food. Each rat was provided with two water bottles on the extreme sides of the cage during the 24-h training phase to adapt the rats to drinking from two bottles. After training, one bottle was randomly switched to contain $0.8 \%$ sucrose solution, a concentration shown in preliminary studies to provide a robust but not maximal sucrose preference (Slattery and Cryan, unpublished), midway through the light cycle (12:00-13:00), and $24 \mathrm{~h}$ later, the bottles were reversed to avoid perseveration effects. After another $24 \mathrm{~h}$, the $0.8 \%$ sucrose solution bottle was replaced with water, and drinking was measured for $24 \mathrm{~h}$ before one of the two bottles was removed from the cage. The use of a 48-h testing period allowed us to preclude any effects of neophobia, artefactual bias toward any one side and perseveration effects. Further, it provides information regarding long-term access to a rewarding stimulus.

Effect of olfactory bulbectomy on cocaine-induced hyperactivity

Two days after the conclusion of the sucrose preference, rats were assessed for locomotor activity during seven consecutive days in a separate room from where they were housed. Locomotor activity was assessed in a novel environment as previously described (Slattery et al. 2005a). An activity monitor consisting of a black and white video camera was mounted in the top-centre of an enclosure $(60 \times 40 \times 50 \mathrm{~cm})$, whereby a cage $(55 \times 33 \times$ $19 \mathrm{~cm}$ ) was positioned in the enclosure. Each second, a single video frame was acquired with a highly accurate, programmable, monochrome frame grabber board (Data Translation, Marlboro, MA, USA; type DT3155). Using inhouse developed software, digitized pixels of two successive frames were compared, and the total number of pixels with altered intensity was counted (independently for pixels with increased and decreased intensity). This allowed the detection of the animal's position within the cage (the centre of the pixels with decreased intensity, because animals were dark compared with background). All test sessions were performed during the early phase of the light cycle (between 08:00-12:00).

Locomotor activity was assessed during a 90-min period, including a 30 -min habituation period before vehicle $(0.9 \%$ saline solution) or cocaine $(10 \mathrm{mg} / \mathrm{kg})$ injection $(1 \mathrm{ml} / \mathrm{kg}$ i.p. $)$. At the end of the 90-min test period, rats were returned to their home-cages. After the 7-day repeated cocaine administration period, rats were weighed daily in their home-cage, and 17 days later, all rats were subjected to a challenge dose of cocaine $(5 \mathrm{mg} / \mathrm{kg} ; 1 \mathrm{ml} / \mathrm{kg}$ i.p.). Similar to the previous cocaine injections, rats were habituated to the environment for $30 \mathrm{~min}$ before injection of cocaine, and the activity was monitored for another $60 \mathrm{~min}$.

\section{ICSS electrode implantation}

Rats were anaesthetized with an isoflurane-oxygen vapor mixture (1-3\% isoflurane, oxygen flow rate $450-$ $500 \mathrm{CCM}$ ) and secured in a stereotaxic frame (TSE Systems). The rats were prepared with a stainless steel bipolar electrode with a diameter of $0.25 \mathrm{~mm}$ (MS303/2, Plastics One) cut to $11 \mathrm{~mm}$ in length into the medial 
forebrain bundle at the level of the posterior lateral hypothalamus (AP-0.5 mm from bregma; $\mathrm{ML} \pm 1.7 \mathrm{~mm}$; DV $-8.3 \mathrm{~mm}$ from dura with the incisor bar at $+5 \mathrm{~mm}$ above the interaural line). The electrode was anchored to four stainless steel skull screws (Plastics One) using dental acrylic. Animals were allowed to recover for at least 7 days before undergoing ICSS training.

\section{ICSS apparatus}

The experimental apparatus consisted of eight Plexiglas chambers $(30.5 \times 30 \times 17 \mathrm{~cm}$; Med Associates $)$ encased in sound-attenuated Coulbourn boxes. The chamber consisted of a stainless steel grid floor and a metal wheel manipulandum located on one of the shorter walls, which required a $0.2-\mathrm{N}$ force to rotate it a quarter turn. Optical sensors determined when the wheel rotated $90^{\circ}$. Gold-contact swivel commutators (SL2C two-channel commutator, Plastics One), and bipolar leads (305-305 TT(CS), Plastics One) connected the animals to a constant current stimulator (Stimtek 1200, San Diego Instruments, San Diego, CA), which in turn was controlled via a DOS-based program (term52) on a PC. The stimulation parameters, data collection and all programming functions were controlled by the computer program.

\section{ICSS procedure}

A discrete-trial ICSS procedure was used (for details see Cryan et al. 2003; Markou and Koob 1992, 1993; Slattery et al. 2005b) that provided current-intensity thresholds, a measure of reward (Kornetsky and Esposito 1979; Markou and Koob 1992, 1993).

Training phase A separate group of rats from the sucrose preference and cocaine-induced hyperactivity experiments were trained on a fixed ratio 1 schedule of reinforcement to turn the wheel $90^{\circ}$, which resulted in the delivery of a train of electrical pulses (typically for a naïve rat comprising of a 100 -ms train of pulses of $150 \mu \mathrm{A}$ with an inter-pulse interval of $0.1 \mathrm{~ms}$ ). After successful acquisition of this reinforcement schedule (two sessions of 100 reinforcers in less than $5 \mathrm{~min}$ ), the outside of discrete-trial procedure was initiated. Each trial was initiated by the delivery of a noncontingent electrical stimulus that was determined to be rewarding for the rats during the initial screening phase on ICSS. The rat had $7.5 \mathrm{sec}$ to respond to receive a second electrical stimulus, identical in all parameters to the noncontingent stimulus. After the rat's response or after the 7.5 sec response window, whichever occurred first, an intertrial interval was initiated that varied in duration from 7.5-12.5 sec. Responding during the intertrial interval postponed the initiation of the next trial by $12.5 \mathrm{sec}$.
After successful training in the above procedure, the current intensity of the non-contingent stimuli, as well as that of the subsequent earned stimuli, was systematically varied, changing $10 \mu \mathrm{A}$ at a time. Current intensities were initially decreased until the animal was no longer responding for two successive stimulus intensities, then increased until the animal responded for two successive stimulus presentations, and then decreased again until the subject did not respond for two successive current intensities. This sequence was repeated twice resulting in three descending and three ascending series, with descending and ascending series alternating. There were three trials within each current intensity. Responses in two out of three trials at a given current intensity were considered a positive response, while no responses in two out of the three trials were considered a negative response (for further details of the procedure, see Markou and Koob, 1992, 1993).

Mean ICSS response thresholds For each test session, six thresholds were calculated, and the mean value was taken as the mean session threshold. The average of the ascending or descending thresholds were calculated separately, as it has been shown previously that certain manipulations can preferentially affect one or other of these measures (Fibiger and Phillips 1981).

ICSS response latency The latency of response was measured, which comprised of the interval between the initiation of the non-contingent stimulus and the operant response of the rat. An average was calculated for each ICSS session to provide a mean latency response time. After 2 to 4 weeks of daily testing, this threshold is generally stable (less than $10 \%$ standard deviation from the previous 3 days), at which time, manipulations could begin.

Extra responses Additional $90^{\circ}$ turns of the wheel manipulandum during the $2 \mathrm{~s}$ immediately after a positive response (a response during the $7.5 \mathrm{sec}$ response window) were counted as extra responses. The total extra responses per session were calculated.

Time-out responses Responses on the wheel manipulandum after the end of the $7.5 \mathrm{sec}$ response window and during the time-out interval were counted as time-out responses. The total number of time-out responses per session were calculated.

Effect of olfactory bulbectomy on ICSS responding

Once stable ICSS baseline thresholds were achieved, sham or olfactory bulbectomy surgery was performed as described above. The day after surgery, rats were returned to the ICSS chamber and their responding assessed daily for 
13 or 14 days. As ICSS responding may become unstable and drift when animals are not presented with their daily ICSS session, it was important to continue the testing of the animals from the day after surgery. It has been reported that some of the behavioural alterations post-bulbectomy only emerge 2 weeks after surgery (see Kelly et al. 1997). However, given the well-documented cognitive impairments after bulbectomy, it would not be possible to wait 2 weeks, as it would have been impossible to distinguish such cognitive deficits from any potential alterations in reward given that the performance of the operant requires instrumental learning ability. As there is no sensitization to the ICSS procedure, we will be able to document any alteration in reward function after bulbectomy in a temporal manner where we hypothesized that maximal impairments would arise 14 days after surgery. Hence, it was important to continue ICSS threshold assessment to prevent thresholds from drifting if a delay is introduced and to assess possible development of anhedonic effects during the days post-surgery. On the 14th (four sham-operated rats and two OB rats) or 15th (ten sham-operated rats and seven OB rats) day post-surgery, all responses had returned to presurgery baseline levels between groups. At this point, animals were administered $7.5 \mathrm{mg} / \mathrm{kg}$ cocaine $(1 \mathrm{ml} / \mathrm{kg}$, i. p.) $10 \mathrm{~min}$ before the ICSS test to examine responding after a psychostimulant challenge. The dose of cocaine was selected from previous studies showing a robust facilitation of ICSS responding at this dose of cocaine (Mague et al. 2005; Slattery et al. 2005b).

\section{Olfactory bulbectomy verification}

At the completion of the behavioural testing, all rats were killed and their brains were dissected to assess the validity of the surgery. A correct bulbectomy was defined as the absence of the bilateral olfactory bulbs without damage to the frontal cortex. Subjects with incomplete or unilateral bulb removal and/or cortical damage were excluded from analysis. Two rats in the ICSS experiment and two rats in the sucrose/cocaine experiment were excluded on this basis.

\section{Data analyses}

Repeated measures analysis of variance (ANOVA) was carried out on all variables in the cocaine-induced hyperactivity and basal ICSS experiments. For ICSS, thresholds on the ascending series, thresholds on the descending series, latency, time-out responses and extra responses were expressed as a percentage of the baseline values assessed during the 3 days before surgery. Assessment of the sucrose preference and cocaine facilitation of ICSS responding test was performed using a Student's $t$ test. Any overall statistical differences, which were set at $p<0.05$, were further analysed using Fisher's least significant difference post hoc. All data were analysed using the SPSS v12 software statistical package.

\section{Results}

Olfactory bulbectomy effects on sucrose preference and cocaine-induced hyperactivity

\section{Body weight}

Olfactory bulbectomy resulted in an acute weight loss after surgery [day $\times$ group $F(42,820)=2.048, p<0.001]$. Posthoc analysis demonstrated that bulbectomized rats significantly lost weight in the first 4 days after surgery compared with sham-operated controls. Thereafter, bulbectomized rats gained weight, but at a slower rate than sham controls (data not shown).

\section{Sucrose preference}

Sucrose preference is frequently used as a measure of anhedonia in rodents (Craft and Devries 2006; El Yacoubi et al. 2003; Grippo et al. 2006; Strekalova et al. 2004; Willner 1997). At the beginning of the sucrose preference test (i.e. after 14 days recovery from surgery) there was no longer a significant difference in body weight gain observed between the groups [weight gain: sham, $48.12 \pm 5.47 \mathrm{~g}$; OB, $34.1 \pm 8.1 \mathrm{~g} ; t(20)$ n.s.]. During the initial training phase, to accustom the rats to drinking from two bottles (both filled with water), no significant difference was observed in water intake between sham and $\mathrm{OB}$ rats $[t(20)=-0.281$, n.s.]. Total sucrose intake during the subsequent 48 -h presentation of $0.8 \%$ sucrose solution or water did not differ between the groups $[t(20)=0.927$, n.s.]. Similarly, no difference in total water intake during this time was observed $[t(20)=$ $-1.158, \mathrm{n} . \mathrm{s}$.]. When the percent sucrose preference was assessed, analysis revealed no significant difference in preference between sham-operated controls and $\mathrm{OB}$ rats $[t(20)=0.903$, n.s. $]$ (see Fig. 1).

\section{Novelty and cocaine-induced hyperactivity}

One of the most reliable and widely used behavioural measures of the OB syndrome is hyperactivity in a novel environment. Statistical analyses were performed on the baseline (i.e. initial $30 \mathrm{~min}$ in locomotor chamber) data and cocaine-induced activity (i.e. 30 to $90 \mathrm{~min}$ in locomotor chamber) with day representing the within-subjects variable. Baseline activity was shown to be significantly greater 
Fig. 1 The effect of surgery on total water, total sucrose and percent sucrose preference variables during the 48-h sucrose preference paradigm and water intake during the 24-h training phase. Data represent mean \pm SEM. Student's $t$ test was performed between the two groups on the variables. There were no differences in any variable assessed
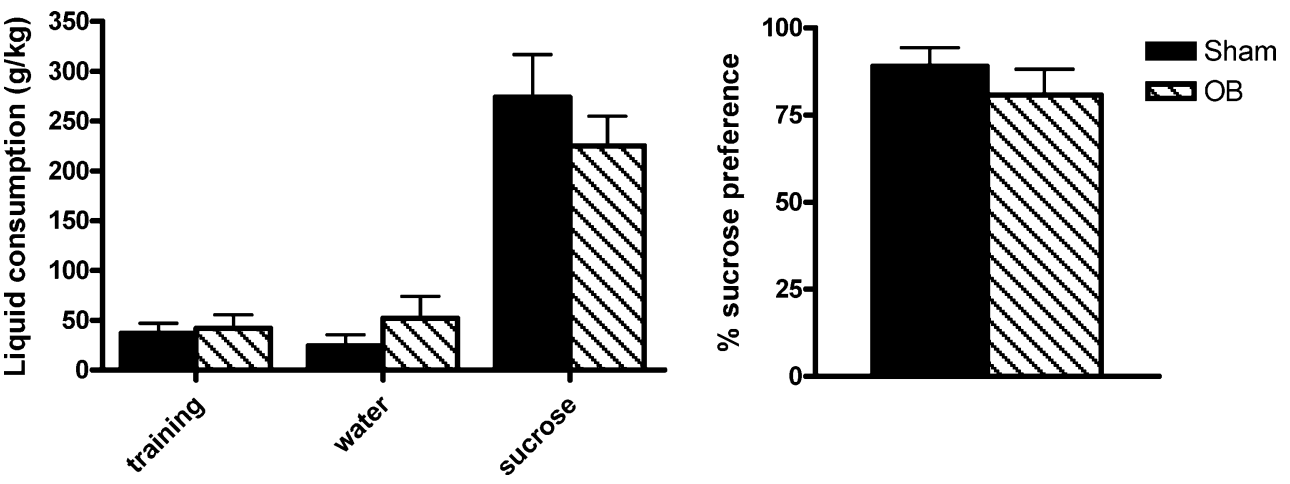

in olfactory bulbectomized rats compared with sham controls [main effect of surgery; $F(1,18)=5.5, p<0.05$ ]. Cocaine treatment also affected baseline activity [main effect of treatment; $F(1,18)=4.496, p<0.05$ ], but there was no interaction effect $[F(1,18)=1.664$, n.s.]. Further analyses revealed a day $\times$ surgery interaction effect $[F$ $(7,126)=4.772, p<0.001]$, but no day $\times$ treatment interaction effect $[F(7,126)=1.942$, n.s.]. Post-hoc analyses demonstrated that on day 1 and on the cocaine challenge day, bulbectomized rats exhibited hyperlocomotion compared with sham controls (Fig. 2).

As expected, cocaine administration $(10 \mathrm{mg} / \mathrm{kg})$ caused a hyperlocomotor effect in both sham and bulbectomized rats [main effect of treatment; $F(1,17)=34.357, p<0.001$ ]. Additionally, a significant effect of surgery on cocaineinduced activity was observed [surgery; $F(1,17)=6.12$, $p<0.05]$, and there was a significant surgery $\times$ treatment interaction $[F(1,17)=4.794, p<0.05]$. Further analyses revealed a significant day $\times$ treatment interaction $[F$ $(7,119)=5.127, p<0.001]$, but no day $\times$ surgery interaction $[F(7,119)=0.824$, n.s. $]$ or day $\times$ surgery $\times$ treatment interactions $[F(7,119)=1.329$, n.s. $]$. Post hoc analyses revealed that cocaine induced hyperactivity on days 2 to 7 in sham controls compared with vehicle administration. Similarly, cocaine was shown to induce hyperactivity in olfactory bulbectomized rats compared with vehicle administration at all time-points, except from the initial exposure to cocaine (Fig. 2).

Separate statistical analyses were performed for each day individually, with time (10-min activity bins) comprising the within-subjects variable. Significant ANOVA effects of time and treatment were observed on each individual day, whereas surgery effects were observed on day 4 and the challenge day. Furthermore, significant surgery $\times$ treatment interaction effects could be demonstrated on the challenge day $[F(1,18)=12.293, p<0.01]$. Post-hoc analyses, were appropriate after a significant overall ANOVA effect, revealed significant effects of both treatment and surgery on locomotor activity, with bulbectomized rats displaying hyperactivity in the novel environment and also in response to cocaine administration both during the repeated administration regimen and to the challenge dose (Fig. 3).

Olfactory bulbectomy effects on intracranial selfstimulation responding

When stable 3-day ICSS threshold baselines were achieved, rats were randomly allocated to sham or olfactory bulbectomy surgery after the final day's ICSS session. Olfactory bulbectomy resulted in significant changes to all parameters measured during ICSS sessions. During the initial 4 days, a subset of bulbectomized rats did not complete two consecutive discrete-trial series to obtain daily threshold values (six on day 1, five on day 2, three on day 3 and one on day 4) and were not included in statistical analyses for thresholds (A to C). However, these animals did complete a sufficient number of reinforced trials to include in response latency and extra response analyses. Similarly, time-out responses were monitored and included in analyses for all days. Thereafter, all rats responded during their daily ICSS session.

\section{Body weight}

As seen in experiment 1, olfactory bulbectomy resulted in a moderate yet significant weight loss, which was maximal 3 days after surgery [repeated measures: surgery $F(1,15)=37.365, p<0.001$; surgery $\times$ day $F(18,270)=$ $19.196, p<0.001]$. Post-hoc analysis revealed that olfactory bulbectomized rats gained less weight over the entire duration of the ICSS experiment, although this appeared to be due to the initial weight loss (Fig. 4).

\section{Thresholds}

Mean ICSS threshold were significantly elevated after bulbectomy [main effect of surgery $F(1,21)=14.385$, $p<0.001$; main effect of day $F(13,298)=10.141, p<0.001$; 
Fig. 2 Effect of bulbectomy on baseline and cocaine-induced locomotor activity in the rat. $A-H$ represent days 1 to 7 and the challenge day baseline locomotor activity i.e. the total locomotion in the initial 30-min testing, respectively. $A^{\prime}-H^{\prime}$ represent days 1 to 7 and the challenge day of total cocaineinduced locomotor activity (60 $\mathrm{min})$, respectively. Two-way repeated-measures ANOVA followed by Fisher's post-hoc was performed. An asterisk indicates $p<0.05$, two asterisks indicate $p<0.01$ and three asterisks indicate $p<0.001$ compared with relevant surgery group. Number symbol indicates $p<0.05$ and two number symbols represent $p<0.01$ compared with relevant treatment group
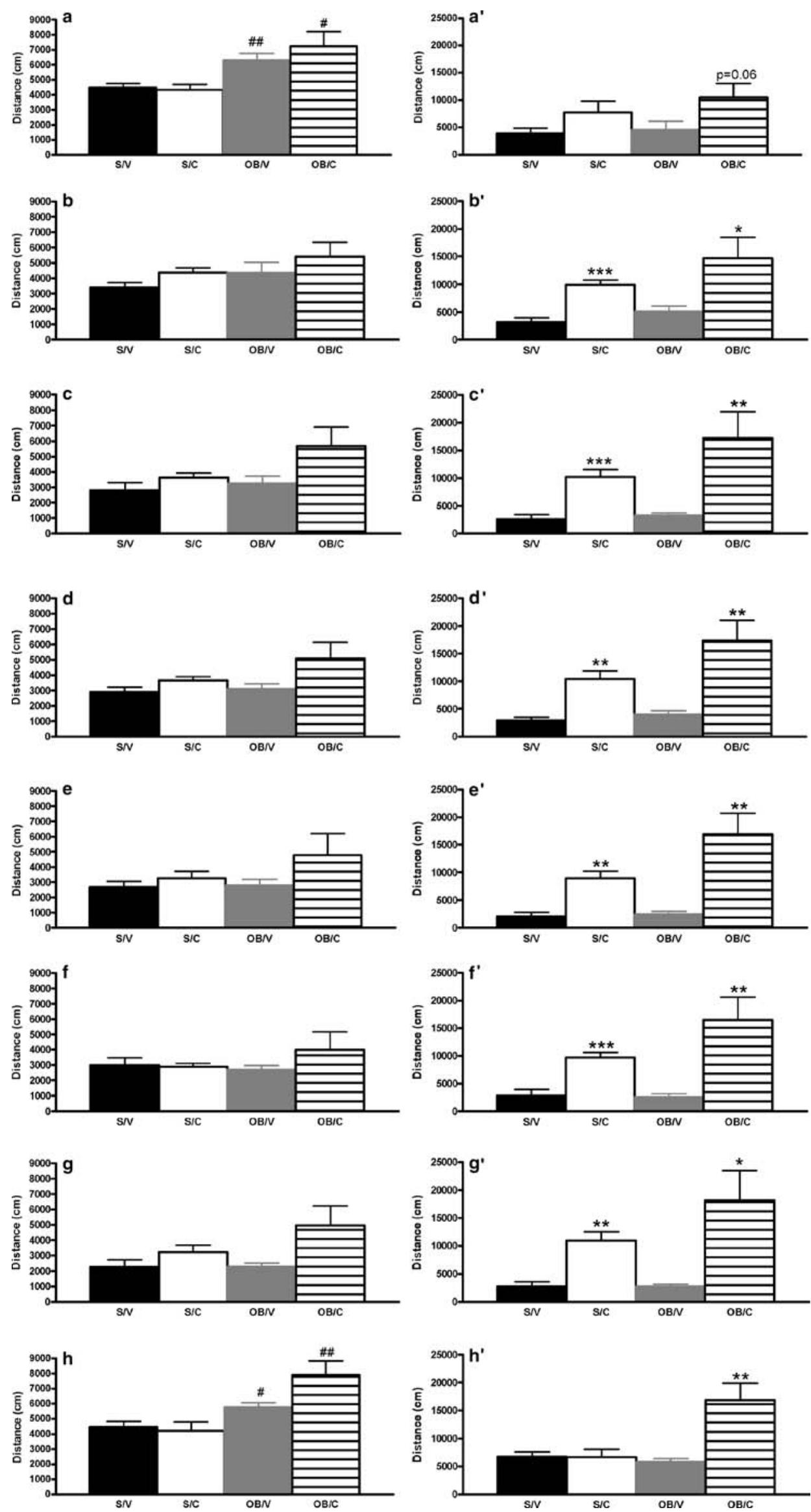

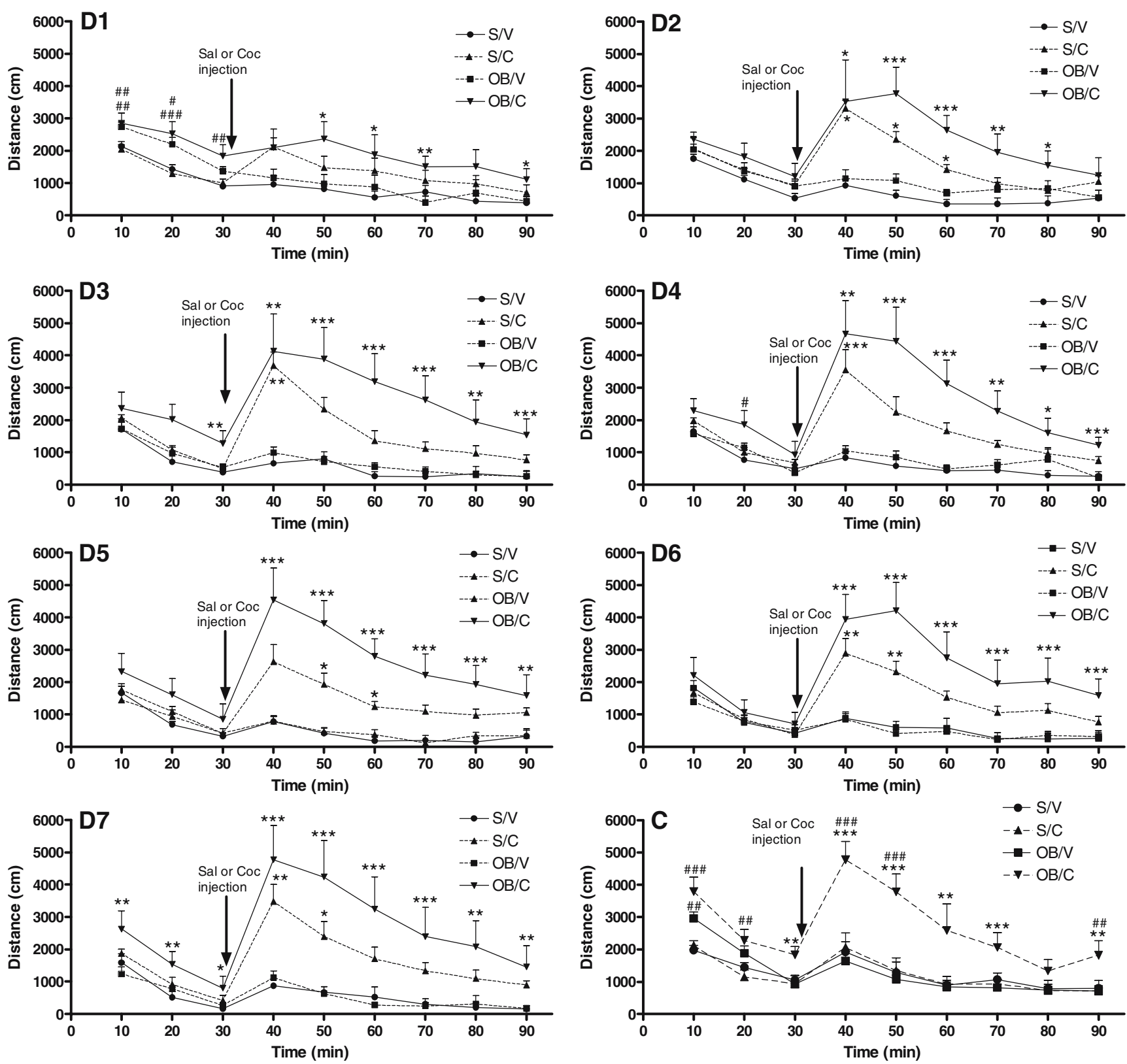

Fig. 3 The effect of olfactory bulbectomy on cocaine-induced hyperlocomotion and cocaine challenge. $D 1-D 7$ and $C$ represent day $1-7$ and challenge day 10-min activity bins, respectively. Data represent mean \pm SEM. Two-way repeated-measures ANOVA followed by Fisher's post hoc was performed for each dataset. An asterisk

indicates $p<0.05$, two asterisks indicate $p<0.01$ and three asterisks indicate $p<0.001$ compared with relevant surgery group. Number symbol indicates $p<0.05$, two number symbols indicate $p<0.01$ and three number symbols indicate $p<0.001$ compared with relevant treatment group

interaction effect of surgery $\times$ day $F(13,298)=7.812$, $p<0.001]$. Post-hoc analysis revealed that bulbectomy resulted in an initial elevated mean threshold, which normalized over the course of testing (by day 8; Fig. 5a). As has been shown previously, manipulations can selectively affect either the ascending or descending reward thresholds derived from the ICSS procedure (Fibiger and Phillips 1981). Thus, we investigated if this was the case after

bulbectomy. As can be seen in Fig. $5 \mathrm{~b}$ and c, when the mean threshold was broken down into ascending and descending means, differences between the groups were also observed. Ascending mean threshold values were significantly increased by bulbectomy surgery [main effect of surgery $F(1,21)=$ $14.697, p<0.001$; main effect of day $F(13,298)=13.053$, $p<0.001$; interaction effect of surgery $\times$ day $F(13,288)=$ $8.137, p<0.001]$, as were descending mean threshold 


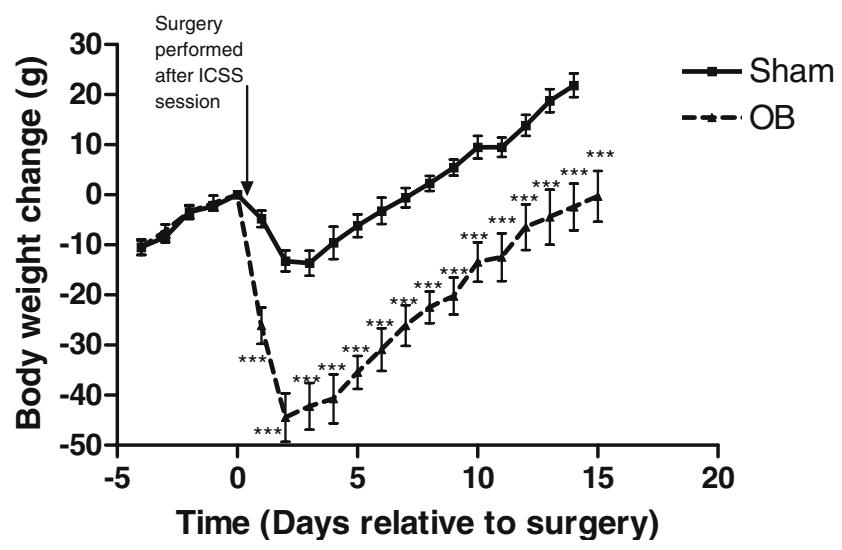

Fig. 4 The effect of olfactory bulbectomy on body weight of rats tested in the ICSS procedure. Data represent mean \pm SEM body weight change (in g) compared with pre-surgery weight. Two-way ANOVA followed by Fisher's post-hoc was performed. Three asterisks represent $p<0.001$ compared with sham control values [main effect of surgery $F(1,21)=10.7, p=0.004$; main effect of day $F(13,298)=10.411, p<0.001$; interaction effect of surgery $\times$ day $F(13,295)=6.268$, $p<0.001]$. Post-hoc analysis revealed that bulbectomized rats exhibited elevated ascending and descending mean thresholds during the initial days after surgery (Fig. $5 \mathrm{~b}$ and c).

\section{Latency}

OB also altered the response latency [main effect of surgery $F(1,21)=0.081$, n.s.; main effect of day $F(13,312)=$ 4.092, $p<0.001$; interaction effect: surgery $\times$ day $F(13$, $312)=2.246, p=0.01]$. Further analysis showed that bulbectomized rats displayed an increase in latency compared with sham-operated controls and with their own presurgery baselines (Fig. 5d).
Fig. 5 The effects of olfactory bulbectomy on ICSS parameters. Olfactory bulbectomized rats (filled triangle) exhibit alteration in a mean threshold, b ascending threshold, c descending threshold, $\mathbf{d}$ response latency, e time-out responses and $\mathbf{f}$ extra responses compared with sham-operated controls (filled square). Data represent mean \pm SEM values compared with the 3-day presurgery mean value. Two-way RM ANOVA followed by Fisher's post-hoc test was performed for each dataset. An asterisk represents $p<0.05$, two asterisks indicate $p<0.01$ and three asterisks indicate $p<0.001$ compared with sham operated controls
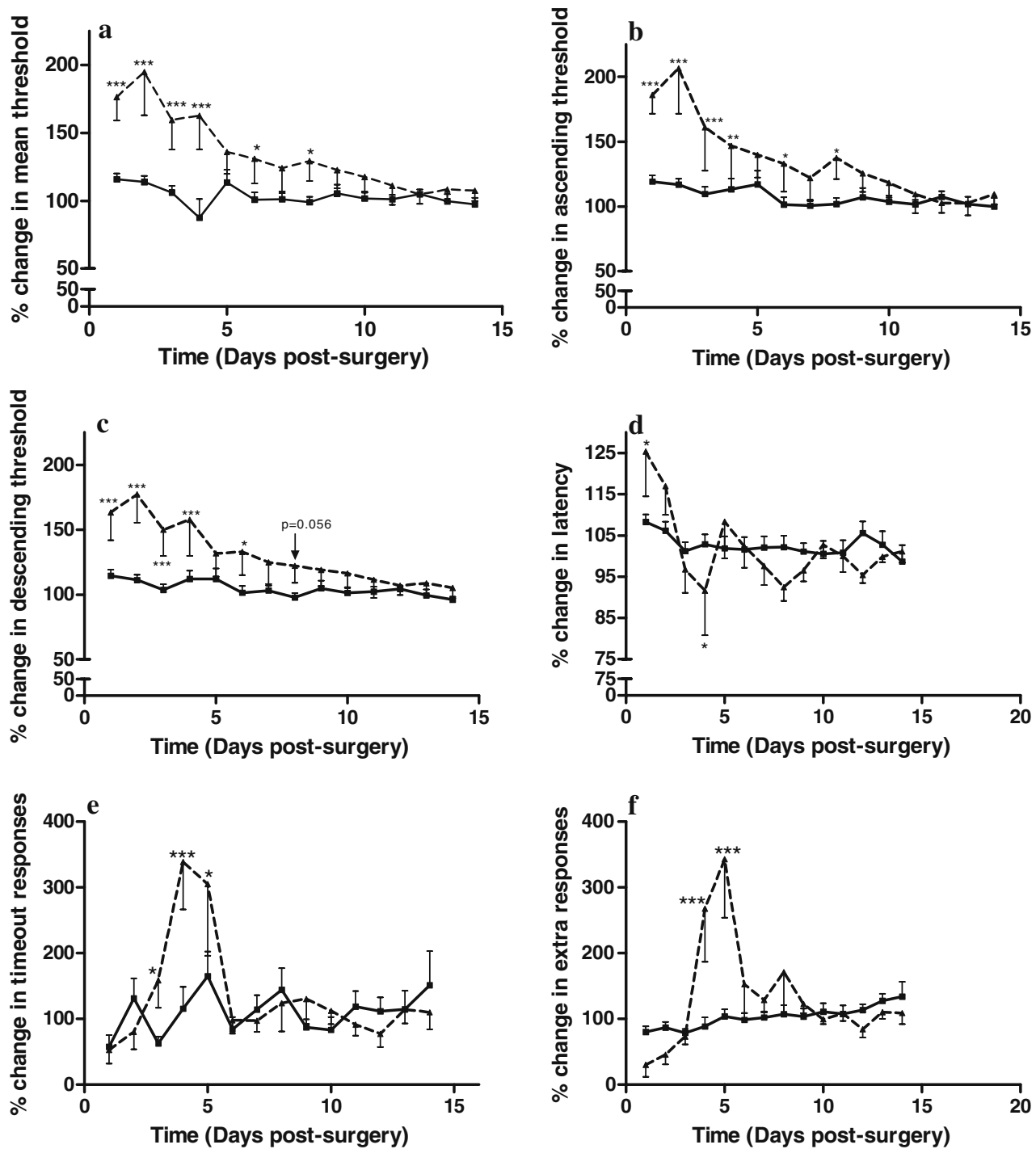


\section{Extra responses and time-out responses}

Both these measures were altered by surgery [extra responses, main effect of surgery $F(1,21)=5.622, p<0.05$; main effect of day $F(13,310)=6.787, p<0.001$; interaction effect: surgery $\times$ day $F(13,310)=6.196, p<0.001$; timeout responses, main effect of surgery $F(1,21)=1.299$, n.s.; main effect of day $F(13,311)=4.991, p<0.001$; interaction effect of surgery $\times$ day $F(13,311)=2.977, p<0.001]$. Post-hoc analyses revealed a trend towards an initial decrease in both responses followed by a significant increase before normalization in bulbectomized rats (see Fig. 5e and f).

\section{Effects of acute cocaine on ICSS responding in $O B$ and sham animals}

In response to an acute administration of cocaine $(7.5 \mathrm{mg} / \mathrm{kg}$ i.p. $10 \mathrm{~min}$ before ICSS session), no significant difference was observed between the two groups (Fig. 6). However, there was a tendency for bulbectomized rats to exhibit an increase in the number of time-out responses $(p=0.068)$.

\section{Discussion}

The present studies demonstrate that olfactory bulbectomy induces pronounced deficits in brain reward function as assessed using ICSS, which are not long-lasting and normalize within 14 days. However, bulbectomized animals have persistent alterations in the locomotor response to the psychostimulant cocaine. Interestingly, the preference for sucrose was not altered by bulbectomy. Additionally, the ICSS threshold-lowering effect of cocaine administration was not altered in comparison with control rats.

Despite its use as an animal model of depression for more than 30 years, there is scant knowledge regarding the alterations that removal of the olfactory bulbs has on brain reward function. This is surprising, given the fact that anhedonia is one of the core symptoms of depression, and
Fig. 6 The effects of olfactory bulbectomy on cocaine-induced alterations in ICSS parameters No effect of olfactory bulbectomy was seen in any parameter a mean threshold, $\mathbf{b}$ ascending threshold, c descending threshold, $\mathbf{d}$ response latency, e timeout responses and $\mathbf{f}$ extra responses. Data represent mean \pm SEM values compared with the 3-day pre-surgery mean value. Student's $t$ test was performed for each dataset
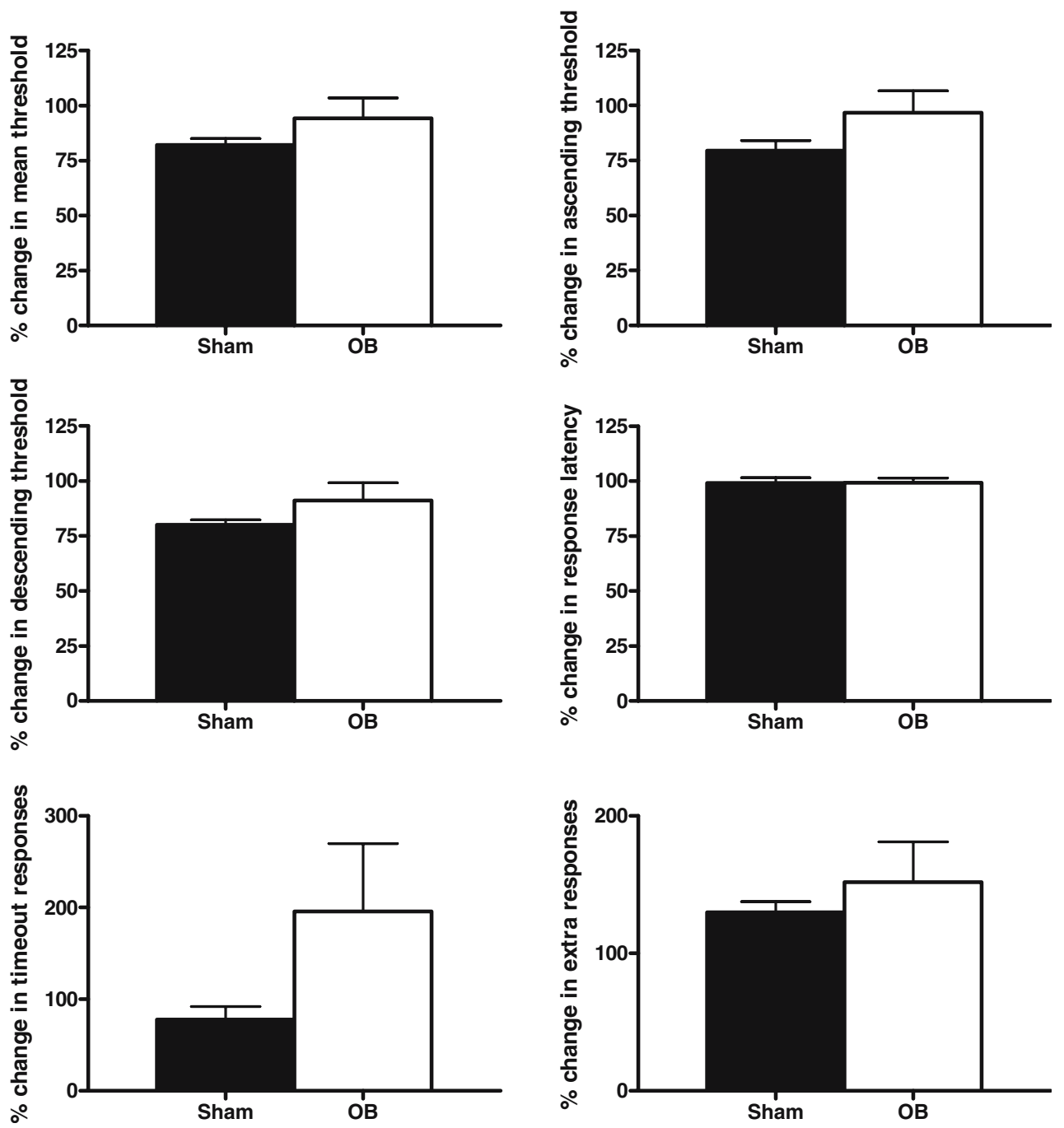
anhedonic-like behaviour has been widely utilized in other animal models of depression, such as chronic mild stress (Willner 1997). An animal, when given a free choice, will preferentially consume a sweet sucrose solution over water, and this can be used to gain insight into the perceived reward an animal can obtain. In this paper, we demonstrate that 2 weeks after olfactory bulbectomy, a time when the behavioural and neurochemical alterations caused by the manipulation are at their zenith (Harkin et al. 2003; Kelly et al. 1997; Song and Leonard 2005), no difference in sucrose preference is observed between surgical groups (Fig. 1). Furthermore, no significant effect was observed on water or sucrose intake. These results suggest that under baseline conditions, with no effort required to obtain a reward, bulbectomy does not affect the brain reward system that underlies the intake of a palatable solution. Previous studies have shown that bulbectomy reduced sucrose preference (Primeaux et al. 2003) or had no effect in male animals but did modify responding in female rats (Stock et al. 2000). However, the former study must be treated with caution, as only the statistical significance was provided in the manuscript and not the raw data. Taken together with the present study, these results suggest that bulbectomy does not cause a robust shift in the rewarding properties of sucrose but suggest that females may be more susceptible to alterations in perceived reward to a palatable solution after bulbectomy.

Although sucrose preference can provide insight into brain reward system functioning, administration of a psychostimulant dopaminergic probe can reveal changes which occur after a challenge to the system (Naranjo et al. 2001). Many drugs of abuse, for example, have been demonstrated to increase dopamine release in the nucleus accumbens, and administration of dopaminergic antagonists can reduce the rewarding properties of such drugs. Masini et al. (2004) demonstrated that under basal conditions, bulbectomized rats displayed increased dopamine release, as measured by microdialysis, in both the dorsal and ventral striatum. This effect is consistent with findings indicating that bulbectomy results in increased locomotor activity in an open field (Chambers et al. 2004; Cryan et al. 1998, 1999; Masini et al. 2004), as locomotor activity is critically dependent on activation of dopaminergic neurotransmission (Kuczenski et al. 1983). Therefore, we assessed challenging the brain reward system with daily administration of cocaine and compared locomotor activity between bulbectomized and control rats.

As has been previously described, bulbectomized rats display hyperactivity in a novel environment (Figs. 2 and 3). This increased ambulation had returned to the level of the sham-operated controls after $30 \mathrm{~min}$, at which time point, the rats received a cocaine or saline injection. Cocaine administration resulted in hyperactivity in both sham and olfactory bulbectomized rats, which was prolonged in bulbectomized rats compared with their respective controls (Figs. 2 and 3). Hyperactivity is a well-characterized response to cocaine administration, and a previous study has shown that bulbectomized rats have a greater ambulation to cocaine than sham-operated controls (Chambers et al. 2004). In the present study, we extended these findings to demonstrate that this effect persisted during the 7-day cocaine administration period and after a final 8th challenge injection of cocaine after a 17-day withdrawal period.

Interestingly, on the 7th day of testing, the bulbectomy cocaine treatment group displayed a greater hyperactivity during the habituation period compared with controls (Fig. 3). This effect suggests that the repeated cocaine administration resulted in persistent alterations to the dopamine system. Studies in naïve animals have shown that those displaying greater locomotor activity also exhibit increased dopamine release in the striatum (Rouge-Pont et al. 1993). We also demonstrate that bulbectomized rats display a significantly greater locomotor response to a cocaine challenge given subsequent to a withdrawal period from the continuous administration regime. This finding lends further support to a persistent alteration in the mesolimbic system after the repeated administration of cocaine in bulbectomized animals. These findings are also in agreement with previously reported studies, which show that bulbectomized rats acquire self-administration of low amphetamine doses faster than controls (Holmes et al. 2002). Analogously, recent findings from human studies have shown persistent alterations in the brain reward system in depressed patients, which engender them hypersensitive to the administration of drugs of abuse. For example, depression severity was closely correlated with the rewarding effects of D-amphetamine $(30 \mathrm{mg} / \mathrm{kg}$ p.o.) administration, with mild/moderate depressives reacting similarly to controls, but severely depressed patients exhibiting a much greater reaction (Naranjo et al. 2001). Similarly, two studies using dextroamphetamine demonstrated hypersensitivity in severely depressed patients (Tremblay et al. 2002; Tremblay et al. 2005). Therefore, the findings in the current study of cocaine-induced hyperactivity in bulbectomized rats are comparable and suggest further study of the underlying circuitry involved in this phenomenon. Further, given the marked co-morbidity between drug dependence and depression, our findings are in agreement with the hypothesis that there are shared underlying neurobiological substrates between both disorders. Whereas it is evident that the locomotor effects of stimulant drugs and their reinforcing effects are not homologous, locomotor activity is critically dependent on activation of dopaminergic neurotransmission (Kuczenski et al. 1983). A pivotal role of ventral tegmental area 
dopaminergic neurons in mediating the hyperlocomotor effects of psychostimulants has been described (Kalivas and Stewart 1991). Nonetheless, further investigation of the effects of bulbectomy in animal models of reward and addiction is necessary.

It is believed that major depression leads to less reinforcement to reward-related cues and has been shown to be associated with reduced emotional responsiveness (Pizzagalli et al. 2005; Shestyuk et al. 2005; Surguladze et al. 2004). Therefore, given that a rewarding stimulus is associated with a positive reinforcement, ICSS responding provides a unique tool with which to concomitantly study positive reinforcement with an accurate, quantifiable measurement of the brain reward system. The results show that there is a dramatic anhedonic-like effect of bulbectomy during the first week after surgery, as it resulted in threshold elevations (Fig. 5a-c), reminiscent of that seen after withdrawal from various psychostimulant drugs (Barr and Markou 2005; Cryan et al. 2003). Nonetheless, such a time frame is not sufficient to initiate a chronic regimen of antidepressant medication and therefore weakens the utility of the paradigm as a model for screening novel antidepressant drugs. Furthermore, as this anhedonic-effect was present during the initial recovery phase from the surgery, we cannot rule out the influence of physically removing the olfactory bulbs (see Fig. 4). Despite the fact that a sham-operated control group was utilized, this group, by necessity, does not undergo the same degree of invasive surgery as the bulbectomy group. Nevertheless, it should be noted that the threshold elevating effects of olfactory bulbectomy persisted longer than the increase in response latency seen after the same manipulation. This observation is significant, as it has been shown that manipulations that affect the motor capabilities of the animal increase response latencies, decrease extra and time-out responses, but have no effect on thresholds (Markou and Koob 1992). Thus, the increase (rather than decrease) in extra and time-out responses early after the olfactory bulbectomy also argues against possible motor-impairing effects of olfactory bulbectomy that may have interfered with responding in the ICSS task.

Moreover, it is also possible that the initial anhedoniclike effect observed after bulbectomy could be the result of a cognitive deficit. The increased number of time-out and extra responses observed after bulbectomy may give some credence to this supposition. However, these behavioural responses could also reflect the increase in impulsivity and agitation previously reported in both bulbectomy (Lumia et al. 1992) and depressed patients (Arango et al. 2002). Furthermore, such alterations in behaviour are probably driven by changes in the serotonergic system as opposed to other central reward systems (Hasegawa et al. 2005; Lumia et al. 1992; Song and Leonard 2005; van der Stelt et al. 2005). This dissociation is further supported by the fact that the increases observed in these parameters follow a markedly different temporal pattern to that of the elevated thresholds, further supporting the concept that these parameters are functionally distinct.

Finally, to assess the perceived rewarding value of an acute administration of a drug of abuse in the olfactory bulbectomy model of depression, the ICSS thresholdlowering properties of this drug were examined. When stable ICSS reward thresholds had returned after surgery, cocaine was administered $10 \mathrm{~min}$ before the ICSS session. As previously demonstrated, cocaine resulted in a robust threshold-lowering response in control rats (Slattery et al. 2005b) and although there was a trend toward an attenuated threshold-lowering effect in bulbectomized rats, this did not reach significance (Fig. 6). This is in stark contrast to the findings of accentuated cocaine responses on hyperlocomotor activity in bulbectomized rats. However, separation between locomotor and rewarding effects of cocaine has previously been demonstrated in rats (Slattery et al. 2005b). Furthermore, bulbectomy has previously been demonstrated to reduce the expression of cocaine-induced place preference (Calcagnetti et al. 1996), lending further support for the dissociation between locomotor and rewarding effects of cocaine.

The only ICSS parameter analysed which differed between the groups was a strong trend toward an increase in time-out responses $(p=0.06)$. This suggests that bulbectomized rats may be more impulsive after administration of a drug of abuse. As impulsivity is largely under the regulation of the serotonergic system (Brunner and Hen 1997), this result further reinforces the dissociation between the neural basis of these responses, which is probably due to the well-documented alterations in the serotonergic system in bulbectomized animals (Hasegawa et al. 2005; van der Stelt et al. 2005) and the altered anhedonia-related effect.

In conclusion, the present study demonstrates an anhedonic-like phenotype after olfactory bulbectomy under basal conditions which is normalized within 2 weeks of further ICSS testing. Additionally, we show that an acute challenge with cocaine provides the same level of reward as assessed with ICSS, whereas cocaine induces a more pronounced hyperactivity in bulbectomized rats compared with sham-operated controls. This study demonstrates the utility of studying singular symptom clusters of major depression in animal models. Although the olfactory bulbectomy model mimics numerous aspects of depression seen in patients, this manipulation does not appear to induce the long-lasting alterations to the brain reward system under basal conditions. Therefore, whereas bulbectomy gives significant insights into certain aspects of 
depression and serves to be an appropriate inducing factor for certain endophenotypes such as cognitive deficits (Kelly and Leonard 1993; Redmond et al. 1994), alterations in cell proliferation (Keilhoff et al. 2006), alterations in neurogenesis (Jaako-Movits and Zharkovsky 2005), neuroendocrine dysfunction (Kelly et al. 1997), amygdala alterations (Song and Leonard 2005), alterations in neuroimmune function (Connor et al. 2000) and hypofunctionality of the serotonergic system (van der Stelt et al. 2005), it appears unlikely to give such information regarding anhedonia. Taken together, these results suggest that bulbectomized rats do display alterations in brain reward function, but these are not long-lasting and not amenable to the study of pharmacological intervention. However, given that bulbectomized animals are hypersensitive to drugs of abuse, future studies will address whether bulbectomy may be an appropriate inducing factor for the development of animal models of co-morbid depression and drug dependence.

Acknowledgement The authors would like to thank Dr Cedric Mombereau, Hugo Buerki and Stefan Imobersteg for their technical assistance and Mr. Mike Arends for editorial assistance. This work was supported by National Institutes of Mental Health/National Institute on Drug Abuse Grant U01 MH69062.

\section{References}

Arango V, Underwood MD, Mann JJ (2002) Serotonin brain circuits involved in major depression and suicide. Prog Brain Res 136:443-453

Barr AM, Markou A (2005) Psychostimulant withdrawal as an inducing condition in animal models of depression. Neurosci Biobehav Rev 29:675-706

Brunner D, Hen R (1997) Insights into the neurobiology of impulsive behaviour from serotonin receptor knockout mice. Ann NY Acad Sci 836:81-105

Calcagnetti DJ, Quatrella LA, Schechter MD (1996) Olfactory bulbectomy disrupts the expression of cocaine-induced conditioned place preference. Physiol Behav 59:597-604

Chambers RA, Sheehan T, Taylor JR (2004) Locomotor sensitization to cocaine in rats with olfactory bulbectomy. Synapse 52:167175

Connor TJ, Harkin A, Kelly JP, Leonard BE (2000) Olfactory bulbectomy provokes a suppression of interleukin-1beta and tumour necrosis factor-alpha production in response to an in vivo challenge with lipopolysaccharide: effect of chronic desipramine treatment. Neuroimmunomodulation 7:27-35

Craft TK, Devries AC (2006) Role of IL-1 in poststroke depressivelike behaviour in mice. Biol Psychiatry 60:812-818

Cryan JF, Holmes A (2005) The ascent of mouse: advances in modelling human depression and anxiety. Nat Rev Drug Discov 4:775-790

Cryan JF, Mombereau C (2004) In search of a depressed mouse: utility of models for studying depression-related behaviour in genetically modified mice. Mol Psychiatry 9:326-357

Cryan JF, McGrath C, Leonard BE, Norman TR (1998) Combining pindolol and paroxetine in an animal model of chronic antidepressant action - can early onset of action be detected? Eur J Pharmacol 352:23-28
Cryan JF, McGrath C, Leonard BE, Norman TR (1999) Onset of the effects of the 5-HT1A antagonist, WAY-100635, alone, and in combination with paroxetine, on olfactory bulbectomy and 8OH-DPAT-induced changes in the rat. Pharmacol Biochem Behav 63:333-338

Cryan JF, Markou A, Lucki I (2002) Assessing antidepressant activity in rodents: recent developments and future needs. Trends Pharmacol Sci 23:238-245

Cryan JF, Hoyer D, Markou A (2003) Withdrawal from chronic amphetamine induces depressive-like behavioural effects in rodents. Biol Psychiatry 54:49-58

Drevets WC (2001) Neuroimaging and neuropathological studies of depression: implications for the cognitive-emotional features of mood disorders. Curr Opin Neurobiol 11:240-249

El Yacoubi M, Bouali S, Popa D, Naudon L, Leroux-Nicollet I, Hamon M, Costentin J, Adrien J, Vaugeois JM (2003) Behavioural, neurochemical, and electrophysiological characterization of a genetic mouse model of depression. Proc Natl Acad Sci USA 100:6227-6232

Fibiger HC, Phillips AG (1981) Increased intracranial self-stimulation in rats after long-term administration of desipramine. Science 214:683-685

Geyer MA, Markou A (2000) Animal models of psychiatric disorders. In: Watson S (ed) Psychopharmacology: the fourth generation of progress. Lippincott, Williams and Wilkins, Philadelphia

Gould TD, Gottesman II (2006) Psychiatric endophenotypes and the development of valid animal models. Genes Brain Behav 5:113119

Grippo AJ, Beltz TG, Weiss RM, Johnson AK (2006) The effects of chronic fluoxetine treatment on chronic mild stress-induced cardiovascular changes and anhedonia. Biol Psychiatry 59:309316

Harkin A, Kelly JP, Leonard BE (2003) A review of the relevance and validity of olfactory bulbectomy as a model of depression. Clin Neurosci Res 3:253-262

Hasegawa S, Watanabe A, Nguyen KQ, Debonnel G, Diksic M (2005) Chronic administration of citalopram in olfactory bulbectomy rats restores brain 5-HT synthesis rates: an autoradiographic study. Psychopharmacology (Berl) 179:781-790

Hasler G, Drevets WC, Manji HK, Charney DS (2004) Discovering endophenotypes for major depression. Neuropsychopharmacology 29:1765-1781

Holmes PV, Masini CV, Primeaux SD, Garrett JL, Zellner A, Stogner KS, Duncan AA, Crystal JD (2002) Intravenous self-administration of amphetamine is increased in a rat model of depression. Synapse 46:4-10

Jaako-Movits K, Zharkovsky A (2005) Impaired fear memory and decreased hippocampal neurogenesis following olfactory bulbectomy in rats. Eur J Neurosci 22:2871-2878

Kalivas PW, Stewart J (1991) Dopamine transmission in the initiation and expression of drug-and stress-induced sensitization of motor activity. Brain Res Brain Res Rev 16:223-244

Keilhoff G, Becker A, Grecksch G, Bernstein HG, Wolf G (2006) Cell proliferation is influenced by bulbectomy and normalized by imipramine treatment in a region-specific manner. Neuropsychopharmacology 31:1165-1176

Kelly JP, Leonard BE (1993) Dexamethasone suppression of corticosterone secretion in the olfactory bulbectomized rat. Neuropsychopharmacology 9:S137-S138

Kelly JP, Wrynn AS, Leonard BE (1997) The olfactory bulbectomized rat as a model of depression: an update. Pharmacol Ther 74:299 316

Kessler RC, Berglund P, Demler O, Jin R, Merikangas KR, Walters EE (2005) Lifetime prevalence and age-of-onset distributions of DSM-IV disorders in the National Comorbidity Survey Replication. Arch Gen Psychiatry 62:593-602 
Kornetsky C (2004) Brain-stimulation reward, morphine-induced oral stereotypy, and sensitization: implications for abuse. Neurosci Biobehav Rev 27:777-786

Kornetsky C, Esposito RU (1979) Euphorigenic drugs: effects on the reward pathways of the brain. Fed Proc 38:2473-2476

Kuczenski R, Leith NJ, Applegate CD (1983) Striatal dopamine metabolism in response to apomorphine: the effects of repeated amphetamine pretreatment. Brain Res 258:333-377

Lumia AR, Teicher MH, Salchli F, Ayers E, Possidente B (1992) Olfactory bulbectomy as a model for agitated hyposerotonergic depression. Brain Res 587:181-185

Mague SD, Andersen SL, Carlezon WA Jr (2005) Early developmental exposure to methylphenidate reduces cocaine-induced potentiation of brain stimulation reward in rats. Biol Psychiatry $57: 120-125$

Markou A, Koob GF (1992) Construct validity of a self-stimulation threshold paradigm: effects of reward and performance manipulations. Physiol Behav 51:111-119

Markou A, Koob GF (1993) Intracranial self-stimulation thresholds as a measure of reward. In: Saghal A (ed) Behavioural neuroscience: a practical approach. IRL, Oxford, pp 93-115

Markou A, Kosten TR, Koob GF (1998) Neurobiological similarities in depression and drug dependence: a self-medication hypothesis. Neuropsychopharmacology 18:135-174

Masini CV, Holmes PV, Freeman KG, Maki AC, Edwards GL (2004) Dopamine overflow is increased in olfactory bulbectomized rats: an in vivo microdialysis study. Physiol Behav 81:111-119

Murray CJ, Lopez AD (1997) Alternative projections of mortality and disability by cause 1990-2020: global burden of disease study. Lancet 349:1498-1504

Naranjo CA, Tremblay LK, Busto UE (2001) The role of the brain reward system in depression. Prog Neuro-psychopharmacol Biol Psychiatry 25:781-823

Nestler EJ (2005) Is there a common molecular pathway for addiction? Nat Neurosci 8:1445-1449

Pizzagalli DA, Jahn AL, O'Shea JP (2005) Toward an objective characterization of an anhedonic phenotype: a signal-detection approach. Biol Psychiatry 57:319-327

Primeaux SD, Wilson MA, Wilson SP, Guth AN, Lelutiu NB, Holmes PV (2003) Herpes virus-mediated preproenkephalin gene transfer in the ventral striatum mimics behavioural changes produced by olfactory bulbectomy in rats. Brain Res 988:43-55

Redmond AM, Kelly JP, Leonard BE (1994) Effect of paroxetine and fluvoxamine on behavioural changes in a number of paradigms in the olfactory bulbectomized rat model of depression. Journal Serotonin Res 1:199-205

Rouge-Pont F, Piazza PV, Kharouby M, Le Moal M, Simon H (1993) Higher and longer stress-induced increase in dopamine concentrations in the nucleus accumbens of animals predisposed to amphetamine self-administration. A microdialysis study. Brain Res 602:169-174

Sheline YI, Mittler BL, Mintun MA (2002) The hippocampus and depression. Eur Psychiatr 17(Suppl 3):300-305
Shestyuk AY, Deldin PJ, Brand JE, Deveney CM (2005) Reduced sustained brain activity during processing of positive emotional stimuli in major depression. Biol Psychiatry 57:1089-1096

Slattery DA, Desrayaud S, Cryan JF (2005a) GABAB receptor antagonist-mediated antidepressant-like behaviour is serotonindependent. J Pharmacol Exp Ther 312:290-296

Slattery DA, Markou A, Froestl W, Cryan JF (2005b) The GABAB receptor-positive modulator GS39783 and the GABAB receptor agonist baclofen attenuate the reward-facilitating effects of cocaine: intracranial self-stimulation studies in the rat. Neuropsychopharmacology 30:2065-2072

Song C, Leonard BE (2005) The olfactory bulbectomised rat as a model of depression. Neurosci Biobehav Rev 29:627-647

Stock HS, Ford K, Wilson MA (2000) Gender and gonadal hormone effects in the olfactory bulbectomy animal model of depression. Pharmacol Biochem Behav 67:183-191

Strekalova T, Spanagel R, Bartsch D, Henn FA, Gass P (2004) Stressinduced anhedonia in mice is associated with deficits in forced swimming and exploration. Neuropsychopharmacology 29:2007-2017

Surguladze SA, Young AW, Seniour C, Brebion G, Travis MJ, Phillips ML (2004) Recognition accuracy and response bias to happy and sad facial expressions in patients with major depression. Neuropsychology 18:212-218

Touzani K, Velley L (1998) Electrical self-stimulation in the central amygdaloid nucleus after ibotenic acid lesion of the lateral hypothalamus. Behav Brain Res 90:115-124

Tremblay LK, Naranjo CA, Cardenas L, Herrmann N, Busto UE (2002) Probing brain reward system function in major depressive disorder: altered response to dextroamphetamine. Arch Gen Psychiatry 59:409-416

Tremblay LK, Naranjo CA, Graham SJ, Herrmann N, Mayberg HS, Hevenor S, Busto UE (2005) Functional neuroanatomical substrates of altered reward processing in major depressive disorder revealed by a dopaminergic probe. Arch Gen Psychiatry 62:1228-1236

van der Kooy D, Fibiger HC, Phillips AG (1977) Monoamine involvement in hippocampal self-stimulation. Brain Res 136:119-130

van der Stelt HM, Breuer ME, Olivier B, Westenberg HG (2005) Permanent deficits in serotonergic functioning of olfactory bulbectomized rats: an in vivo microdialysis study. Biol Psychiatry 57:1061-1067

Willner P (1997) Validity, reliability and utility of the chronic mild stress model of depression: a 10-year review and evaluation. Psychopharmacology (Berl) 134:319-329

Wise RA (2002) Brain reward circuitry: insights from unsensed incentives. Neuron 36:229-240

Wise RA (2005) Forebrain substrates of reward and motivation. J Comp Neurol 493:115-121

Wise RA, Bozarth MA (1982) Action of drugs of abuse on brain reward systems: an update with specific attention to opiates. Pharmacol Biochem Behav 17:239-243

Wise RA, Bozarth MA (1985) Brain mechanisms of drug reward and euphoria. Psychiatr Med 3:445-460 